\title{
A Particle Filtering method for Wireless Sensor Network Localization with an Aerial Robot Beacon
}

\author{
F. Caballero, L. Merino, I. Maza and A. Ollero
}

\begin{abstract}
This paper presents a new method for the 3D localization of an outdoor Wireless Sensor Network (WSN) by using a single flying beacon-node on-board an autonomous helicopter, which is aware of its position thanks to a GPS device. The technique is based on particle filtering and does not require any prior information about the position of the nodes to be estimated. Its structure and stochastic nature allows a distributed computation of the position of the nodes. The paper shows how the method is very suitable for outdoor applications with robotic data-mule systems. The paper includes a section with experiments.
\end{abstract}

\section{INTRODUCTION}

Latest advances in low-power electronics and wireless communication systems have made possible a new generation of devices able to communicate, sense environmental variables and even process this information, the Wireless Sensor Networks (WSNs). In addition, the recent commercialization of these devices has increased the applicability and research efforts in this area.

One of the major challenges for WSN deployment is the localization of the sensor nodes. This localization is essential to all those applications that require spatial mapping of the sensed data for further processing, and is also useful for the overall system performance as the availability of positioning information enable the adoption of low overhead protocols such as geographic-based routing schemes. It has been pointed out [1] that, when the number of sensors is large, the manual deployment and position recording is errorprone and, in many applications, hand-placing the sensor is not an option. Thus, for example, if the sensors are scattered from an airplane, a different localization method should be employed. This is particularly true for networks deployed in emergency response scenarios without preexisting infrastructure, as considered in the AWARE project devoted to the development of a platform for autonomous selfdeploying and operation of wireless sensor-actuator networks cooperating with aerial vehicles.

The Global Positioning System (GPS) provides an immediate solution to the problem of localizing a node in outdoor scenarios. However, having GPS in all the nodes is often not a viable solution in many scenarios, due to its cost,

F. Caballero, I. Maza and A. Ollero are with the University of Seville, Camino de los Descubrimientos s/n, 41092, Sevilla (Spain). caba@cartuja.us.es, imaza@cartuja.us.es, aollero@cartuja.us.es

L. Merino is with Pablo de Olavide University, Carretera Utrera km1, 41013, Sevilla (Spain). lmercab@upo.es

This work is partially supported by the AWARE project funded by the European Commission (IST-2006-33579), and the AEROSENS (DPI-200502293) and SADCON (2005/TEP-375) Spanish projects the associated energy consumption, and its inapplicability in different scenarios.

Outdoor WSN localization begins to be an active research topic where different approaches has been proposed [1], [2], [3], [4]. Most of them are based on the localization of entire network by knowing the position of a small and well distributed set of static nodes, usually called beacon-nodes, with GPS or other positioning devices. Then, the radio interface of the wireless nodes is used to estimate the distance to the beacon-nodes. A pre-calibrated relation between the received signal strength (RSSI) and the distance is used to obtain a low accurate estimation which worsens with the distance from the node to the beacon-nodes. Improvements in the radio interface to increase the directivity [5] or the inclusion of new features like time-of-flight have been proposed [6] but, in general, they lead to a power consumption growth and to a radio coverage reduction. Unfortunately, even having good localized beacon-nodes and a reliable system to propagate this information, the results will be poor if the beacon-nodes are not well distributed in the network.

This paper proposes the use of a mobile node, i. e. a robotic vehicle with GPS, for the localization of the nodes of the network by using the RSSI value to propagate the position information of the robot to the rest of the network. Although the proposed method can be used with any autonomous vehicle, the implementation with autonomous helicopters is proposed in the paper. These vehicles overcome the limitations of accessing with ground vehicles close enough to the static nodes in many scenarios [7]. Furthermore, the use of these aerial vehicles, flying close the ground nodes, favor the propagation of the radio signals by decreasing the effect of obstacles that influence the signal strength.

Particle filtering is used to process the RSSI value in each node in order to localize the nodes of a static wireless network. The method takes into account the uncertainty associated to the RSSI value in order to optimally compute the mean and standard deviation of the localization of the node. In addition, the stochastic nature of the technique allows to extend the classic localization framework, a set of fixed beacon-nodes distributed in the network, to a more flexible one where mobile beacon-nodes are considered.

Initial results on wireless sensor localization using signal strength were conducted by Ladd et al. in [8], where ethernet devices were used to localize and track a human operator inside a building with good results in terms of localization error. Later, mobiles nodes have been considered for outdoor network localization in [1] and [9] by means of stochastic grids and triangulation respectively. In this paper we propose 
the application of particle filters. These filters are more flexible than grids or simple triangulation, as the number of particles can be adjusted depending on the resources and the stochastic nature of the measures can be considered into the estimation process. Also, as it will be seen, the framework presented can localize the nodes in 3D; employing a 3D grid would be too costly for a node in terms of memory requirements.

The proposed method is very suitable for data-mule systems in which the mule is a robot able to self-localize and that carries a node. This node is used to recover the information from the WSN while, at the same time, is employed as a beacon node for network localization.

The paper is structured as follows. Firstly, the approach is outlined in section II. Section III will detail the proposed method to address the localization problem. Finally, the implementation and some experimental results with a real network are shown.

\section{LOCALIZATION FRAMEWORK}

The proposed localization framework is composed by a set of WSN nodes which position is completely unknown and by a moving aerial robot beacon (MARB). The moving robot has attached a positioning device (i.e. GPS) and can move over the area covered by the network.

As mentioned before, the RSSI of the signal received from MARB and its positions are used to estimate the position of the rest of the network nodes. This approach presents the following benefits with respect other techniques as mean or weighted mean triangulation:

- A Bayes filter is employed for the estimation of the localization of the nodes. The estimated position of the nodes will be represented by a probability distribution. This allows to take into account the uncertainty on measures involved in the process, mainly the RSSI/Distance.

- The localization process is based on a mobile aerial robot beacon (MARB), which allows to reduce one of the endemic problems of the RSSI-based localization algorithms: the distortion induced by radio-frequency effects. The chance of measuring the RSSI at different beacon positions and orientations permits automatic detection of outliers and, hence, an improvement in the distance computation.

- It is not required to filter the RSSI measures because they will be automatically weighted by its associated standard deviation, making easier the localization process. Then, it is possible to use the RSSI measures of all the beacons even if they are relatively far from the node.

Thus, it is not strictly required three or more beacons to have an estimation of the position of every node, with the MARB providing information from separate positions. There is not triangulation but an estimation process so that the algorithm can consider hypotheses in which the estimated position is spread over a certain area. This increases the flexibility of the method.

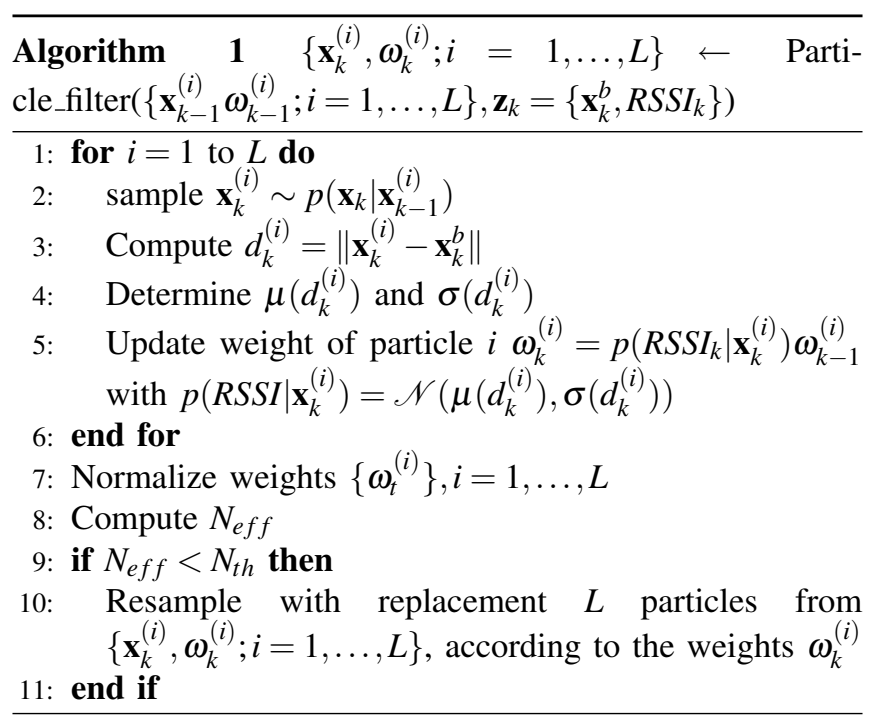

\section{PARTICLE FILTER BASED LOCALIZATION}

The objective of the localization algorithm is to estimate the position of the nodes of the network from the data provided by the MARB. A separated filter per node will be implemented. Then, the state to be estimated consists of the position of the node $\mathbf{x}=\left(\begin{array}{lll}X & Y & Z\end{array}\right)^{T}$. The information about the state will be obtained from the set of measurements $\mathbf{z}_{1: k}$ received up to time $k$. This set of measurements consists of pairs of RSSI and MARB position values $\left\{\mathbf{x}_{k}^{b}, R S S I_{k}\right\}$ (the algorithm considers a moving beacon node, and thus the time subscript for the beacon node position).

In the Bayesian framework employed, the information about the state is represented by the conditional probability distribution $p\left(\mathbf{x}_{k} \mid \mathbf{z}_{1: k}\right)$. This distribution (the posterior) can be estimated online while functioning the network. Therefore, the position will be estimated and updated recursively.

\section{A. Filter Overview}

Particle filtering is a technique for implementing recursive Bayesian filtering by Monte Carlo sampling. The key idea is to represent the posterior density at time $k p\left(\mathbf{x}_{k} \mid \mathbf{z}_{1: k}\right)$ by a set of independent and identically distributed (i.i.d.) random particles $\left\{\mathbf{x}_{k}^{(i)}\right\}$ according to the distribution. Each particle is accompanied by a weight $\omega_{k}^{(i)}$. Sequential observations and model-based predictions will be used to update the weight and particles respectively. See [10] for more details.

Particle Filters allow Bayesian estimation to be carried out approximately but in a structured and iterative manner, that simplifies the implementation on nodes. In general, it is very suitable for non-gaussian stochastic processes with nonlinear dynamics and very useful when the posterior $p\left(\mathbf{x}_{k} \mid \mathbf{z}_{1: k}\right)$ has no parametric form or this form is unknown. Therefore, Particle Filter seems to be a good solution to address the localization problem.

Although there are many possible implementations, in the proposed algorithm the prior probability distribution $p\left(\mathbf{x}_{0}\right)$ is used as the importance (or proposal) distribution to draw the initial set of particles at time 0 , i.e. $\mathbf{x}_{0}^{(i)} \sim p\left(\mathbf{x}_{0}\right)$. Then, these 
particles are recursively re-estimated following the algorithm shown in Algorithm 1.

The next subsections describes the main issues in the actual implementation of the algorithm. As the likelihood function is the core of the algorithm, it is described first. Then the updating step, the prior distribution, the prediction step and the resampling procedure are detailed. Finally, some guidelines for computing the mean and standard deviation in the filter are mentioned.

\section{B. Learning the likelihood function}

The likelihood function $p\left(\mathbf{z}_{k} \mid \mathbf{x}_{k}\right)$ plays a very important role in the estimation process. In this case, this function expresses the probability of obtaining a given RSSI value from a beacon node given the position of the receiving node $\mathbf{x}_{k}$.

Experimental results show that there exists a correlation between the distance to the emitter and the RSSI value, although this correlation decreases with the distance between the two nodes, transmitter and receiver. This is mainly caused by radio-frequency effects such as radio reflection, multi-path or antenna polarization.

The model used here considers that $p\left(\mathbf{z}_{k} \mid \mathbf{x}_{k}\right)$ follows a Gaussian distribution for a given value of $d_{k}$, the distance between the position of the node and the beacon node $\| \mathbf{x}_{k}-$ $\mathbf{x}_{k}^{b} \|:$

$$
R S S I_{k}=\mu\left(d_{k}\right)+\mathscr{N}\left(0, \sigma\left(d_{k}\right)\right)
$$

This section deals with the computation of the relations $\mu\left(d_{k}\right)$ and $\sigma\left(d_{k}\right)$ that relate the received RSSI in one node and the distance to the emitter, which are estimated off-line from a training data set.

A couple of Mica2 Crossbow nodes have been distanced from 0 to 30 meters and the RSSI has been recorded for each distance. This experiment has been repeated with several antenna polarizations. A least squares process was used to compute the $\mu(d)$ and $\sigma(d)$ functions that best fit the set of data. The results are shown in Fig. 1. Notice that the RSSI of these nodes is a dimensionless value from 0 (max power) to 390 (min power). As expected, it can be seen that the standard deviation increase with the distance $d$

This experiment is only carried out for a couple of nodes of the network. Unfortunately, the nodes on a WSN are similar but not exactly the same, and therefore the previous relations should be computed for all the nodes of the network. To avoid this problem the computed standard deviation has been intentionally overestimated in order to include as much nodes as possible. However, this overestimation will increase the time needed to converge to a correct solution in the Particle Filter.

Nevertheless, the experiment data agree with those obtained in [1], where the authors also identify a quasi-gaussian distributions in the relations RSSI vs. distance.

\section{Updating}

Once learned, the functions $\mu(d)$ and $\sigma(d)$ are used online in the estimation process. Each time a new measure is

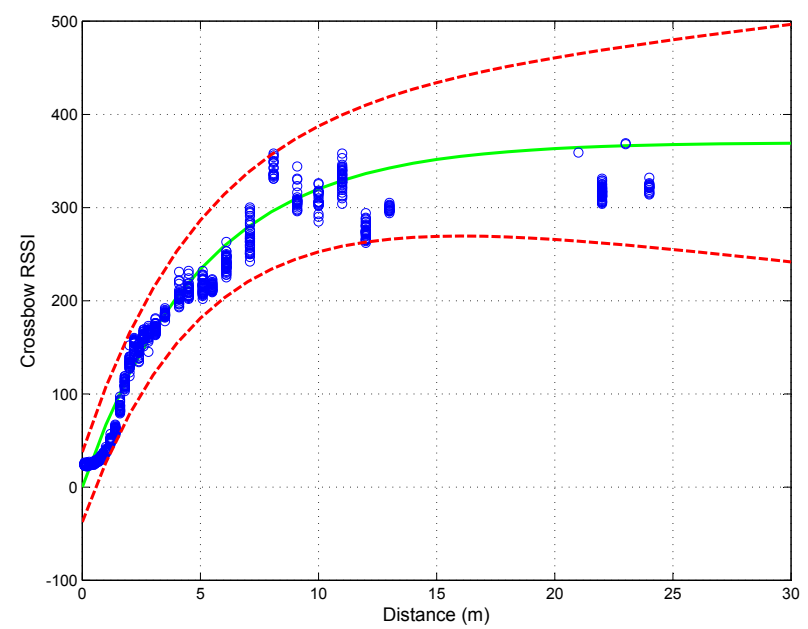

Fig. 1. RSSI-Distance function, $\mu(d)$ and $\sigma(d)$. This function relates the distance between two nodes and the RSSI received in mean and std. deviation. It has been experimentally computed using a large set of couples RSSI/distance. Blue dots: A sub-set of the experimental set of data. Green solid line: Estimated mean $\mu(d)$. Red dashed line: standard deviation confidence interval based on $\sigma(d)$.

received, the weights of the particles are updated considering the likelihood of the received data (lines 3, 4 and 5 of Algorithm 1).

The procedure is as follows. For each particle, the distance $d_{k}^{(i)}=\left\|\mathbf{x}_{k}^{(i)}-\mathbf{x}_{k}^{b}\right\|$ is obtained. From this distance, the mean and variance of the conditional distribution $p\left(\mathbf{z}_{k} \mid \mathbf{x}_{k}^{(i)}\right)$ are obtained, so that $p\left(\mathbf{z}_{k} \mid \mathbf{x}_{k}^{(i)}\right)=\mathscr{N}\left(\mu\left(d_{k}^{(i)}\right), \sigma\left(d_{k}^{(i)}\right)\right)$.

The probability of the actual RSSI value under this distribution is finally employed to update the weight of the particle $\omega_{k}^{(i)}$.

$$
\omega_{k}^{(i)}=\frac{1}{\sigma\left(d_{k}^{(i)}\right) \sqrt{2 \pi}} \exp \left(-\frac{\left(\operatorname{RSSI}_{k}-\mu\left(d_{k}^{(i)}\right)\right)^{2}}{2 \sigma\left(d_{k}^{(i)}\right)^{2}}\right) \omega_{k-1}^{(i)}
$$

\section{Initializing the filter. The prior model}

Up to now, nothing has been commented about the initial distribution from which the particles are drawn.

The filter is initiated when the first message from the MARB is received. In this case, the RSSI distance functions of Fig. 1 are used inversely as in the estimation process. From the RSSI values, an initial distance is estimated, and also a corresponding variance on the distance.

The prior considered is then an uniform distribution on an spherical annulus, in which the inner and outer radius depend on the estimated mean and variance (see Fig. 2). As the number of particles is limited, not all the messages received initiate the filter. Only when a RSSI value corresponding to a variance below a threshold is received, the filter is initiated, in order to have a good resolution (particles per volume unit).

\section{E. Prediction}

The nodes of the WSN are static, so the prediction step might be ommitted (that is, with probability 1 each node is in the same position at time $k$ and $k-1$ ). However, as the 


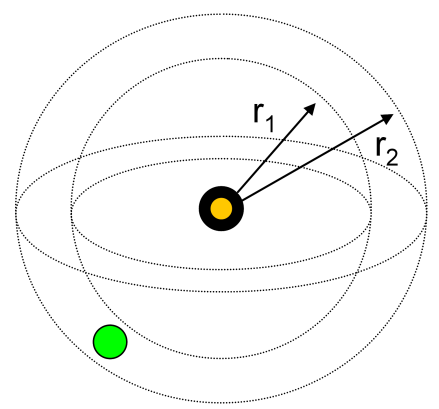

Fig. 2. Prior distribution. The initial samples are drawn from an uniform distribution over an spherical annulus. The inner $\left(r_{1}\right)$ and outer $\left(r_{2}\right)$ radius are a function of the estimated distance from the RSSI and its variance.

resolution of particles over the state space is limited, a random move is added to the particles (step 2 of Algorithm 1), in order to search locally over the position space around the position of the previous time step. Therefore, the prediction model is:

$$
p\left(\mathbf{x}_{k} \mid \mathbf{x}_{k-1}\right)=\mathscr{N}\left(\mathbf{x}_{k-1}, \Sigma_{k-1}\right)
$$

The value of $\Sigma$ depends on the distribution of particles, mainly the density of particles per volume unit.

\section{F. Resampling}

When the filter is running, the weights of the particles with high likelihood will increase, while most of the particles will rest at places of very low likelihood on the state space.

Again, as the number of particles is limited, a resampling step (line 10 of Algorithm 1) is included in order to increase the accuracy of the estimated position, duplicating particles with high weights and eliminating those with very low weights.

In order to overcome some of the known problems with the resampling stage, two additional considerations are taken into account: first, resampling only takes place when the effective number of particles $N_{e f f}$ is below a threshold. The effective number is computed as follows:

$$
N_{e f f}=\left[\sum_{i=1}^{L}\left(\omega_{k}^{(i)}\right)^{2}\right]^{-1}
$$

The threshold is set to the $10 \%$ of the number of particles, so $N_{t h}=0.1 L$.

Second, the algorithm employed for resampling is a low variance sampler. It will allow to spread the particles over the maximum likelihood areas. The algorithm used is the one described in [11] (p. 110).

\section{G. Estimation of mean and std. deviation}

The mean and standard deviation from the filter will be computed as follows:

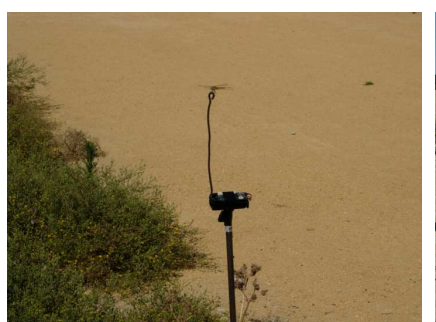

(a)

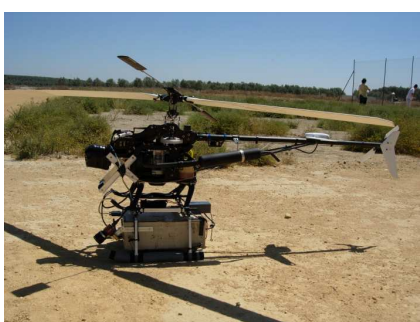

(b)
Fig. 3. Experiment setup. (a): One of the deployed nodes with a dragonfly on the antenna. (b): The UAV. At the back of the electronic box it can be seen the beacon node with its antenna.

$$
\begin{aligned}
\mu_{k} & =\sum_{i=1}^{L}\left[\mathbf{x}_{k}^{(i)} \omega_{k}^{(i)}\right] \\
\sigma_{k}^{2} & =\sum_{i=1}^{L}\left(\mathbf{x}_{k}^{(i)}-\mu_{k}\right)^{2} \omega_{k}^{(i)}
\end{aligned}
$$

One of the benefits of the Particle Filter is that allows to face multi-modal or non-parametric hypothesis. While the posterior distribution will depend on the measures during the transient state, the filter approximately converge to a Normal distribution in the position of the node. It has been considered that the filter converges when $\sigma_{k}$ is below a certain threshold during a period of time. In the implementation the threshold was set to $3 m$ during at least 15 messages.

If the filter converges at time $k_{0}$, the belief on the position of the node can be modeled as a Normal distribution such as $\mathscr{N}\left(\mu_{k_{0}}, \sigma_{k_{0}}\right)$. This allows switching to another filter implementation like Extended Kalman Filter (EKF) or Unscented Kalman Filter (UKF) which will efficiently take into account the gaussian nature of the posterior distribution.

\section{IMPLEMENTATION AND EXPERIMENTAL RESULTS}

In order to test the previous ideas, an experimental setup was conceived. A small WSN composed by 5 nodes was deployed outdoor. The nodes of the network are Mica2 nodes (see Fig. 3.a), with the following characteristics:

- Atmega128L microcontroller at $7.3728 \mathrm{Mhz}$

- Chipcon CC1000 FSK radio transceiver, $900 \mathrm{Mhz}$ band. Up to $4 \mathrm{dBm}$ transmission power. Up to $78.4 \mathrm{kbps} 100$ meter outdoor range.

- Radio programmable for quick software update on deployed networks.

- $512 \mathrm{~KB}$ non-volatile memory for programs and retrieved data.

Before the experiment starts, the position of all the nodes were computed by means of a differential GPS device in order to validate the estimation from the Particle Filter.

In the setup, a node of the network is carried by the HERO 3 autonomous helicopter of the University of Seville (see Fig. 3.b). This vehicle is able to localize itself with high accuracy by using a differential GPS device. The node is connected though a serial port to the onboard computer, so that the UAV 


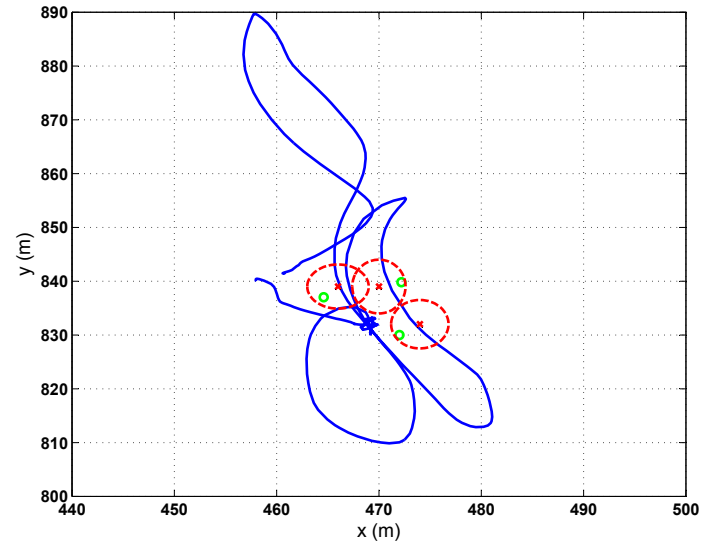

Fig. 4. Estimated position of the nodes versus their position measured employing DGPS. Green dots: real position of the nodes. Red crosses: Estimated position. Red dashed line: standard deviation confidence interval. Solid blue line: UAV trajectory.

position is transmitted to the node. It will act as a MARB for the WSN.

The algorithm proposed in the above section can be implemented in the gateway (laptop) of the WSN. The middleware presented in [12] have been used. Then, the network can be localized as follows:

1) The UAV flies over the WSN.

2) The beacon node onboard the UAV sends a message with its position once per second.

3) When a node receives a beacon message, a message with the position of the beacon and the RSSI on reception is sent to the gateway of the WSN.

4) The information is stored in the gateway and the position of all the nodes are computed by using the above algorithm.

The filter has been setup with 1000 particles per node and non-prior information about the node was assumed. Fig. 4 shows the estimated mean position of the three nodes of the network (the node on board the UAV and the gateway are not considered) and the $3-\sigma$ confidence interval, and the trajectory carried out by the helicopter. It can be seen that the uncertainty in $\mathrm{Y}$ axis is slightly shorter than in $\mathrm{X}$ axis due to the motion of the MARB, which mainly moved over the $\mathrm{Y}$ axis. In fact, the figure shows how the maximum triangulation in $\mathrm{X}$ is about 20 meters while in $\mathrm{Y}$ is about 80 meters.

Detailed information about the estimated position of one of the nodes of the network by using the algorithm of Section III is shown in Fig. 5. It can be seen that the estimations converge to the actual position in the $\mathrm{X}, \mathrm{Y}$ and $\mathrm{Z}$ coordinates. Fig. 6 shows the estimated standard deviation from the particles compared to the error between the estimated mean and the actual position. It can be seen that the estimated deviation from the particles is consistent with the error committed. Notice the hard transitions occurred at messages 60 and 80, it was caused by an automatic resampling step carried out in the particle filter.
The evolution of the Particle Filter is shown in Fig. 7. The initial sphere-like distribution converge gradually to an unimodal distribution approximately centered in the real position of the node.

\section{CONCLUSIONS AND FUTURE WORK}

The paper has presented a particle filter for node position estimation on a WSN. The filter integrates the RSSI measures of the signal received from an aerial robot acting as a beacon node with GPS in order to estimate the position. The experiments have shown the feasibility of the approach. Although the particle filter for each node was run in a laptop in the current implementation, the filter setup was conceived taking into account the processing capabilities of the nodes. Next steps will adapt the presented approach to be implemented inside the nodes.

There are several aspects that will be addressed in the short future. The aerial robot with the beacon is part of the WSN and can be used as a data mule while the positions are being calibrated. Moreover, the current ideas can be used in UAVs capable of deploying nodes in the framework of the AWARE project.

If the path planning capabilities of the mobile robot takes into account the calibration mission, optimal paths from the point of view of network calibration could be devised.

Experiments have shown how the belief on the position of the node can be modeled as a Normal distribution when the Particle Filter converges (see Fig. 7). Future work will consider the particle filter estimation as the initial position of the nodes as in [13]. Then, the estimated mean and covariances will be used in an Unscented Kalman Filter (UKF) [14] that will update this estimation with the measurements not only from the beacon node but from the rest of the network nodes too. The likelihood function of Section III-B will be used to update the sigma-points of the UKF. These filters can be efficiently implemented in the nodes and easily decentralized, so that the information from neighbor nodes can be included in the estimation process.

Finally, in this paper, the likelihood function that relates RSSI and distance was learnt off-line and then maintained fixed. Improvements to re-estimate online the functions by using methods like Expectation-Maximization [15] will be researched.

\section{ACKNOWLEDGMENTS}

The authors thank the valuable help of Pablo Gil and Antidio Viguria during the experiments. The second author also thanks the support provided by the URUS project (IST2006-045062) funded by the European Commission.

\section{REFERENCES}

[1] M. Sichitiu and V. Ramadurai, "Localization of wireless sensor networks with a mobile beacon," in Proc. of the IEEE International Conference on Mobile Ad-hoc and Sensor Systems (MASS), 2004, pp. 174-183.

[2] N. Bulusu, J. Heidemann, and D. Estrin, "GPS-less low-cost outdoor localization for very small devices," IEEE Personal Communications, vol. 7 , no. 5, pp. 28-34, 2000. 

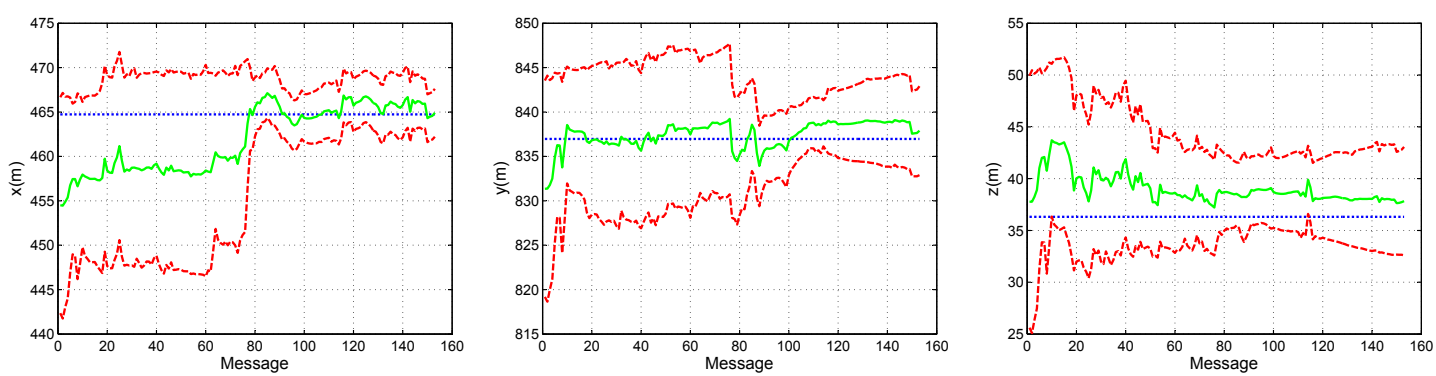

Fig. 5. Estimated node position (green solid), position computed with GPS (blue dash-dotted) and standard deviation confidence interval (red dashed).
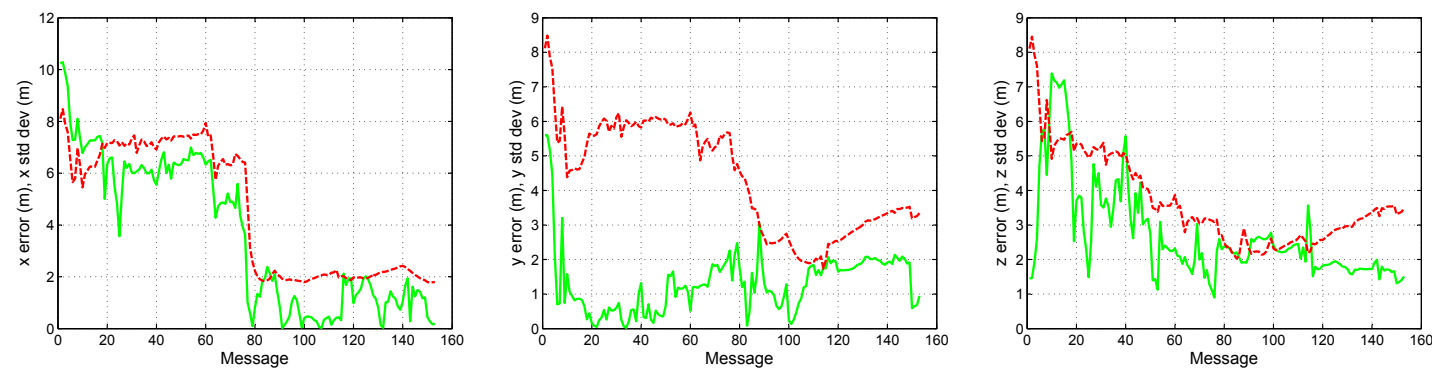

Fig. 6. Standard deviation obtained from the particles (red dashed) and error between the GPS-based position estimation and the mean position computed from the particles (green solid). The estimated distribution is consistent with the values.
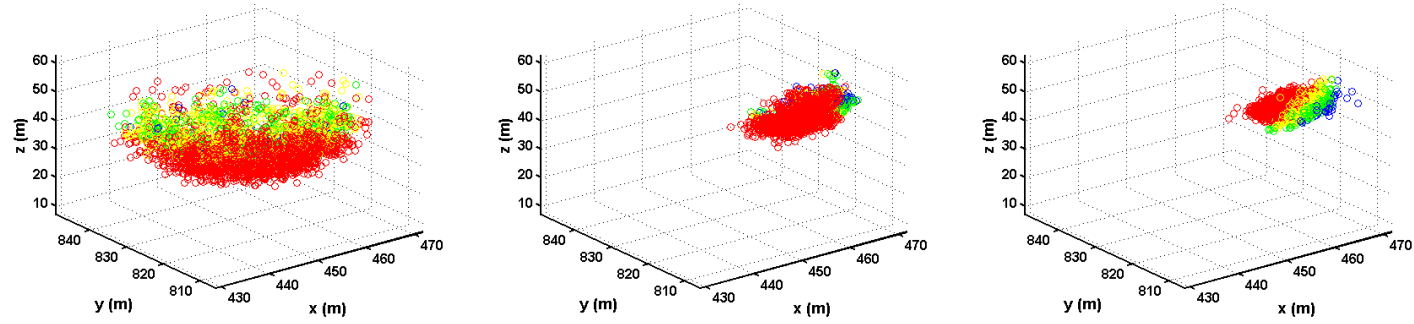

Fig. 7. The particles at three stages. In the first one, it can be identified the sphere-like shape. As more messages are received, the particles concentrate into a unimodal distribution. The color represents the likelihood of the particle: Red between 0 and 0.25 , Yellow between 0.25 and 0.5 , Green between 0.5 and 0.75 , Blue between 0.75 and 1 .

[3] N. Patwari and A. O. Hero, "Using proximity and quantized RSS for sensor localization in wireless networks," in Proc. of the 2nd ACM international conference on Wireless sensor networks and applications, WSNA 2003. New York, NY, USA: ACM Press, 2003, pp. 20-29.

[4] M. Terwilliger, A. G. V. Bhuse, Z. H. Kamal, and M. A. Salahuddin, "A localization system using wireless sensor networks: A comparison of two techniques," in Proc. of the First Workshop on Positioning, Navigation and Communication, Hannover, Germany, March 2004.

[5] S. Yang and H. Cha, "An empirical study of antenna characteristics toward rf-based localization for ieee 802.15.4 sensor nodes," in 4th European conference on Wireless Sensor Networks, EWSN 2007, Delf, The Netherlands, January 2007, pp. 309-324.

[6] K. Whitehouse and D. Culler, "Macro-calibration in sensor/actuator networks," Mobile Networks and Applications, vol. 8, no. 4, pp. 463472, 2003.

[7] A. Ollero and L. Merino, "Control and perception techniques for aerial robotics," Annual Reviews in Control, no. 28, pp. 167-178, 2004, elsevier (Francia).

[8] A. M. Ladd, K. E. Bekris, G. Marceau, A. Rudys, D. S. Wallach, and L. E. Kavraki, "Using wireless Ethernet for localization," in Proceedings of the 2002 IEEE/RSJ International Conference on Intelligent Robots and Systems, IROS 2002, vol. 1, Lausanne, Switzerland,
September 2002, pp. 402-408.

[9] P. Corke, R. Peterson, and D. Rus, "Coordinating aerial robots and sensor networks for localization and navigation," in Proceedings of the 7th International Symposium on Distributed Autonomous Robotic Systems, DARS 2004, Toulouse, France, June 2004, pp. 281-29.

[10] A. Doucet, N. de Freitas, and N. Gordon, Eds., Sequential Monte Carlo Methods in Practice. Springer-Verlag, 2001.

[11] S. Thrun, W. Burgard, and D. Fox, Probabilistic Robotics. The MIT Press, 2005.

[12] P. Gil, I. Maza, A. Ollero, and P. Marron, "Data centric middleware for the integration of wireless sensor networks and mobile robots," in In Review for the 7th IEEE Conference on Mobile Robots and Competitions, Paderne, Algarve, Portugal, 2007.

[13] F. Caballero, L. Merino, P. Gil, I. Maza, and A. Ollero, "A probabilistic framework for entire wsn localization using a mobile robot," Journal of Robotics and Autonomous Systems, 2008, accepted for publication.

[14] S. Julier and J. Uhlmann, "A new extension of the Kalman filter to nonlinear systems," in Proceedings of the 11th Int. Symp. on Aerospace/Defence Sensing, Simulation and Controls, 1997.

[15] R. Neal and G. Hinton, A view of the EM algorithm that justifies incremental, sparse, and other variants. Cambridge, MA, USA: MIT Press, 1999, pp. 355-368. 